References:

[1] Olovnikov A.M, J. Theor. Biol. 41(1):181-190, 1973.

[2] Blackburn E.H., Gall J.G., J. Mol. Biol. 120:33-53, 1978.

[3] Hayflick L, Exp. Cell. Res. 37:614-636, 1965.

[4] Andrews N.P. et al., Gerontology 56(4):390-403, 2010.

[5] Dehbi A. ZA. Et al., Clin. Immunol. 9(12):1193-1204, 2013.

[6] Graakjaer J. et al., Mech. ageing dev., 124(5): 629-640, 2003.

Acknowledgements: The study was funded by RSF (research project No 14-15-00346).

Disclosure of Interest: None declared

DOI: 10.1136/annrheumdis-2017-eular.3649

\section{THU0020 INCREASED EXPRESSION OF CCN4/WISP1 IN OSTEOARTHRITIC ARTICULAR CARTILAGE IS EPIGENETICALLY REGULATED AND DISRUPTS CARTILAGE HOMEOSTASIS}

M. van den Bosch ${ }^{1}$, Y.F. Ramos ${ }^{2}$, W. den Hollander ${ }^{2}$, N. Bömer ${ }^{2}$, R.G. Nelissen ${ }^{3}$, J.B. Bovée ${ }^{4}$, A.B. Blom ${ }^{1}$, I. Meulenbelt ${ }^{2} .{ }^{1}$ Experimental Rheumatology, Radboud university medical center, Nijmegen; ${ }^{2}$ Department of Molecular Epidemiology; ${ }^{3}$ Department of Orthopedics; ${ }^{4}$ Department of Pathology, Leiden University Medical Center, Leiden, Netherlands

Background: Previously, we described increased expression of Wnt-1-induced signaling protein 1 (Wisp1) in murine synovium and cartilage after induction of two experimental osteoarthritis $(\mathrm{OA})$ models. Wisp1 is a downstream target of the canonical Wnt signaling pathway that has been shown to play a pivotal role in the etiopathology of OA. In line with this, we have observed increased breakdown of the articular cartilage after overexpression of Wisp1 in naïve mouse knee joints, whereas Wisp $1^{-1-}$ mice revealed decreased cartilage degeneration in three independent experimental OA models compared to wild type controls. Together, these data indicate a direct correlation between Wisp1 and OA in mice.

Objectives: In the current study we set out to characterize the relation between expression of WISP1 and human OA.

Methods: Articular cartilage from preserved and degenerated $\mathrm{OA}$ areas was collected from 39 Caucasian end-stage OA patients. Cartilage from non-OAdiagnosed individuals was collected after femoral neck fractures. Cartilage degeneration was classified according to the Mankin scoring system. DNA was isolated to determine correlation between WISP1 expression and methylation profiles using Generalized Linear Mixed Model (GLMM). RNA expression levels were determined with microarray analysis and RNA sequencing. Immunohistochemical staining was used to determine WISP1 protein expression. Recombinant WISP1 was added to human chondrocyte microparticles, and cartilage extracellular matrix deposition was determined by measuring cartilage microparticle size and Safranin O/Fast Green staining.

Results: We observed increased WISP1 expression in cartilage of OA patients compared to non-OA-diagnosed controls. Moreover, within OA patients, both WISP1 mRNA and protein expression were significantly increased in OA-affected cartilage compared to preserved regions of the same joint, and WISP1 expression significantly correlated with Mankin score. Interestingly, we found that positional CpG dinucleotides were hypomethylated in cartilage of OA-affected areas as compared to unaffected areas from the same joint, which correlated with increased RNA expression as determined with both microarray analysis and RNA sequencing analysis. Of note, methylation levels of a CpG affecting WISP1 transcription were found to highly significantly correlate to a single nucleotide polymorphism (SNP) at the WISP1 locus. Next, to investigate effects of increased WISP1 levels on chondrocyte microparticles, we added human recombinant WISP1. This resulted in a significantly decreased deposition of cartilage extracellular matrix as reflected by decreased microparticle circumference, and a strongly decreased proteoglycan content, suggesting that increased WISP1 levels are detrimental to cartilage.

Conclusions: The expression of WISP1, both RNA and protein, is increased in OA-affected as compared to preserved articular cartilage. This increased expression is inversely correlated with methylation levels of a positional CpG for WISP1, which was found to be under the influence of a SNP at the WISP1 locus. Together, our results suggest that tight regulation of WISP1 expression via methylation is essential to maintain cartilage homeostasis.

Disclosure of Interest: None declared

DOI: 10.1136/annrheumdis-2017-eular.4010

\section{THU0021 THE ANALYSIS OF PRF1, STX11, UNC13D AND STXBP2 GENE IN SYSTEMIC JUVENILE IDIOPATHIC ARTHRITIS WITH MACROPHAGE ACTIVATION SYNDROME}

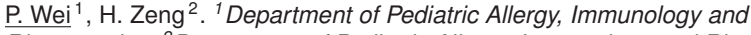
Rheumatolog; ${ }^{2}$ Department of Pediatric Allergy, Immunology and Rheumatology, Guangzhou Women and Children's Medical Center, Guangzhou, China

Objectives: We investigated the clinical characteristics of 34 cases with systemic juvenile idiopathic arthritis (SJIA) and macrophage activation syndrome (MAS) and analyzed PRF1, UNC13D, STX11 and STXBP2 gene so as to figure out the genetic pathogenic mechanism.

Methods: The clinical characteristics of 34 cases with SJIA and MAS were retrospectively analyzed, and the coding sequences of PRF1, UNC13D, STX11 were amplified. DNAMAN software and NCBI BLAST were used to compare sequences. The chi-square test was employed to compare the distribution of allele and genotype frequencies between SLE patients and healthy controls.

Results: Among the 34 cases, $69 \%$ were males, and the average age was 6 years. $85 \%$ (29/34 cases) of the cases underwent genetic testing and four SNPS loci were detected: PRF1 c. $1061 \mathrm{c}>\mathrm{T}$ (rs885822); UNC13D c. $659 \mathrm{c}$ $>$ T (rs3744007); STXBP2 c. $1483 \mathrm{~T}>\mathrm{c}$ (rs10001) and STXBP2 c. $1616 \mathrm{~A}$ $>\mathrm{G}$ (rs6791). Compared with control group, genotype and allele frequencies of PRF1 rs885822 and STXBP2 rs10001 among the MAS cases were of significant difference ( $r$ 885822: allele frequency $2=4.52, p=0.03$; genotype frequency: 2 $=5.52, p=0.02$. rs 10001 : allele frequency $2=21.33, p=0.00$; genotype frequency: $2=19.58, p=0.00)$. There were no significant differences in genotype and allele frequencies of UNC13D rs3744007 and STXBP2 rs6791 between MAS group and control group ( $r s 3744007$ : allele frequency $2=1.89, p=0.17$; genotype frequency: $2=1.59, p=0.45$. rs 6791 : allele frequency $2=1.69, p=0.19$; genotype frequency: $2=2.09, p=0.35$ ). The main characteristics of the cases were persistent fever, progressive hepatosplenomegaly, a sharp decline in blood cells, effusion, significantly increased serum liver enzymes, hyperlipidemia, etc. Some children had mucosal bleeding, neurological dysfunction, etc. More than $82 \%$ children had upper respiratory tract infection before MAS onset. $90 \%$ of children had remission, while three cases developed multiple organ failure and died.

Conclusions: Macrophage activation syndrome is fatal complication caused by immune imbalance. Early detection and treatment is the key to improve the prognosis. The SNP PRF1 rs885822 and STXBP2 rs1001 may be concurrent with the pathogenesis of SJIA-MAS. The SNP UNC13D rs3744007 and STXBP2 rs6791 may not play a role in SJIA-MAS.

Disclosure of Interest: None declared

DOI: 10.1136/annrheumdis-2017-eular.2138

\section{THU0022 REPLICATION ANALYSIS OF GENE-GENE INTERACTION BETWEEN HLA-DQA2 AND HLA-DQB2 VARIANTS IN ITALIAN RHEUMATOID ARTHRITIS PATIENTS}

R. Bassi Andreasi ${ }^{1}$, M.F.J. Khan ${ }^{1}$, E. Galuppi ${ }^{2}$, M. Govoni ${ }^{2}$, M. Rubini ${ }^{1}$ ${ }^{1}$ Biomedical and Specialty Surgical Sciences; ${ }^{2}$ Medical Sciences, University of Ferrara, FERRARA, Italy

Background: Rheumatoid Arthritis (RA) is a complex and multifactorial disease, caused by multiple interactions between genetics and environmental factors. In the context of RA genetics, the Human Leukocyte Antigen (HLA) exerts a primary role. Approximately $90 \%$ of RA patients show alleles at the HLA-DRB1 locus, codifying for 5 Amino Acids, known as "shared epitope" (SE). SE is associated with increased risk to develop RA and more severe phenotype, but it does not entirely explain HLA contribution to disease risk.

Moreover, it is generally accepted that occurrence risk could be determined by specific gene-gene interactions. A study carried out in USA (NARAC) reported a particular interaction between SNPs in the HLA class II region: HLA-DQA2 (rs9275595) and HLA-DQB2 (rs10807113). Results showed 11 fold increased risk to develop RA in patients carrying both variants (Liu et al., 2011), while single variants did not significant contribute to the RA risk (Odds ratio, OR, respectively, 1.6 and 1.0$)$.

Gene-gene interaction between HLA-DQA2 and HLA-DQB2 SNPs is remarkable, considering that glycoproteins codified by these genes combined each other and create a quaternary structure.

Objectives: To replicate NARAC study evidence in Italian population, we genotyped rs9275595 (HLA-DQA2) and rs10807113 (HLA-DQB2) variants using a case/control model.

Methods: The study included a cohort of 316 Italian RA patients and a matched group of 443 healthy control individuals. All subjects were genotyped for HLA-DQA2 T > C (rs9275595) and HLA-DQB2 A > C (rs10807113) using TaqMan technology and Real-Time PCR.

Results: Replication test performed in our Italian RA cohort did not identify significant interaction between two variants analyzed.

However, considering the single variant rs9275595, the results showed a significantly increased risk to develop RA. Taking T/T genotype as the reference, $\mathrm{C} / \mathrm{C}$ homozygotes showed significant association with a 3.82 fold increased risk of RA (OR=3.821 95\% Cl (2.017-8.351) $\mathrm{p}$-value $=0.00063)$. The SNP acted under an additive model: T/C heterozygotes showed significant $\mathrm{OR}=1.988(95 \% \mathrm{Cl}$ $1.458-2.711$, p-value $=0.00002$ )

Conclusions: The results of this study do not confirm the gene-gene interaction previous reported in the NARAC cohort (Liu et al., 2011). However, outcomes suggest that HLA-DQA2 rs9275595 could contribute to RA pathogenesis in the Italian population.

\section{References:}

[1] Liu C, Ackerman HH, Carulli JP. A genome-wide screen gene-gene interactions for rheumatoid arthritis susceptibility. Hum Genet. 2011 May;129(5):473-85. Disclosure of Interest: None declared DOI: 10.1136/annrheumdis-2017-eular.6252 